\title{
Surgical Results and Risk Factors for Recurrence of Lumbar Disc Herniation
}

\author{
Jung Tae Oh, Ki Seok Park, Sung Sam Jung, Seung Young Chung, \\ Seong Min Kim, Moon Sun Park, Han Kyu Kim \\ Department of Neurosurgery, Eulji University College of Medicine, Daejeon, Korea
}

\begin{abstract}
Objective: Recurrent lumbar disc herniation has been defined as disc herniation at the same level, regardless of ipsilateral or contralateral herniation, with a pain-free interval greater than 6 months. The aim of this study is to analyze outcomes and identify the potential risk factors for recurrent lumbar disc herniation.

Methods: The authors retrospectively reviewed the cases of 178 patients who underwent open discectomy for single-level lumbar disc herniation. Visual analogue scales and modified Macnab criteria were used to compare the clinical outcomes between the recurrent group and the non-recurrent group.

Sex, age, discectomy level, degree of disc degeneration, type of disc herniation, pain-free interval after first-operation, smoking status, and trauma were investigated as potential recurrence risk factors.

Results: Of the 178 patients for whom the authors were able to definitely assess symptomatic recurrence status, 18 patients (10.1\%) underwent revision surgery for recurrent disc herniation. The most common level involved was L4-L5 (61\%) and the mean period of time to recurrence was 18.7 months (6-61 months). There were 17 cases of ipsilateral herniation and 1 case of contralateral herniation. The types of herniation for which revision surgery was done were protrusion (3 cases), and transligamentous extrusion (14 cases). There were five excellent, eight good, and two fair results.

Conclusion: Repeated discectomy for recurrent disc herniation produced unsatisfactory outcomes. Factors such as sex, type of disc herniation and traumatic events were found to be significant risk factors.
\end{abstract}

Key Words: Lumbar vertebrae $\cdot$ Discectomy $\cdot$ Recurrence $\cdot$ Risk factors

\section{INTRODUCTION}

A number of surgical techniques for treating disc herniation recently have been developed and applied to patients who do not respond to conservative treatment.

Although various surgical options exist for lumbar disc herniation patients, open discectomy has been proven to have lasting benefits in numerous cases, but with diverse outcomes ${ }^{1,8,28}$.

Some patients suffer from persistent or recurrent radiating pain after open discectomy; this pain can be accompanied by a recurrence of the disc herniation. The reported prevalence of unsatisfactory results after discectomy is between 5\%

- Received: May 11, 2012 • Revised: September 18, 2012

- Accepted: September 25, 2012

Corresponding Author: Ki Seok Park, MD

Department of Neurosurgery, Eulji University College of Medicine, Dunsan2dong, Seogu, Daejeon 302-799, Korea

Tel: +82-42-611-3442, Fax: +82-42-611-3444

Email: KS3432@eulji.ac.kr and $20 \%{ }^{19}$. Recurrent herniation is one of the most common causes for an unsatisfactory outcome following lumbar discectomy and occurs in 5-15\% of patients ${ }^{2,5,17,24,26}$. Recurrent lumbar disc herniation has been defined as disc herniation at the same level after previous discectomy, regardless of ipsilateral or contralateral herniation, with a pain-free interval greater than 6 months $^{26}$.

There have been many studies on recurrent disc herniation reporting diverse risk factors including age, gender, body mass index (BMI), smoking, traumatic events, operation time, level of herniation, type of disc herniation, degree of disc degeneration, and the duration of symptoms ${ }^{5,6,9,17,26}$.

In this study, the authors analyzed surgical outcomes after open discectomy and the risk factors for recurrent disc herniation.

\section{MATERIALS AND METHODS}

In this study, we included 178 patients who had undergone open discectomies for lumbar disc herniation between May 
2006 and December 2009. Hospital records and imaging studies, including magnetic resonance imaging (MRI) of all patients, were retrospectively reviewed. In all patients with new symptoms, MRI images (T1- and T2- weighted sagittal and axial view) were obtained and assessed for recurrent disc herniation. The MRI scans revealed 18 patients who appeared to have recurrent disc herniation at the same level and who were unresponsive to conservative treatment. Eighteen patients underwent reoperation and were reviewed retrospectively during the mean follow-up period of 16.5 months.

The non-recurrent group included 98 patients who had experienced no recurrence of leg pain until the most recent follow-up; the mean follow-up period was 27.9 months.

Visual analogue scales (VAS) and modified Macnab criteria were used to compare the clinical outcomes between the recurrent and the non-recurrent group.

Sex, age, discectomy level, degree of disc degeneration, type of disc herniation, smoking status, and traumatic events were considered risk factors for recurrent lumbar disc herniation. The pain-free interval after the first operation was considered a clinical parameter for the prognosis of recurrent lumbar disc herniation.

The degree of degeneration of the herniated disc was assessed on T2-weighted sagittal sequences. Degeneration of the nucleus pulposus was classified according to the method of Horton and Daftari ${ }^{9)}$, in which a point value is given to the signal intensity of the nucleus (white, 0 points; speckled, 1 point; dark, 2 points) ${ }^{4)}$. In addition, a score was given for the presence of a high-signal intensity line within a disc with reduced signal intensity (absent, 0 points; present, 1 point); this finding is related to the presence of fluid within cystic spaces or fissures in markedly degenerated discs.

Annular degeneration was graded on the basis of the height of the disc (normal or slightly decreased, 0 points; moderately or markedly decreased, 1 point). The overall disc degeneration score was then calculated on a scale of $0-4(0$, no degeneration; 4, marked degeneration) using the sum of the nuclear and the annular scores.

The extent of disc herniation was rated on T1- and T2-weighted images according to the methods of Masaryk et al. ${ }^{14)}$ and Modic et al. ${ }^{16)}$, differentiating among protrusion, extrusion, and sequestration.

On axial and sagittal MRI sequences, the type of disc herniation was defined as "protruded" if there was a focal extension of the disc beyond the posterior margin of the vertebral body, "extruded" if disc tissue had migrated through a defect in the outer annular fibrosus but was connected to the disc, and "free fragment of sequestrated" if the herniated tissue was no longer connected to the disc.

The pain-free interval after first operation in the recurrent group was divided into four groups according to recurrence within the first year, at 1-3 years, at 3-5 years, or more than 5 years post-surgery.

The Mann-Whitney U-test and the Chi-square test were used for statistical analyses; results were considered significant when the probability value ( $\mathrm{p}$-value) was less than 0.05 . All statistical analyses were performed using SPSS Statistics 20.0.

\section{RESULTS}

The patients in the non-recurrent group were older than the patients in the recurrent group at the time of diagnosis (56.6 years versus 46.4 years), but this was not considered statistically significant $(\mathrm{p}>0.05)$.

The recurrent group was composed of 5 men and 13 women; the gender distribution difference was statistically significant $(p$ $<0.05)$.

Seventeen patients from the recurrent group had ipsilateraldisc herniation and one patient had a contralateral disc herniation.

Disc herniation recurred at level L4-5 in 11 patients (61 $\%)$, at L5-S1 in 5 patients (28\%), and at the other levels in 2 patients (11\%; 1 case at L2-3 and 1 case at L3-4). Most herniation recurrences were at the L4-5 level, but the differences in recurrence rates among levels were not statistically significant $(\mathrm{p}>0.05)$.

Most patients in the two groups had a score of 3 points on the MRI disc-degeneration scale. The average score was 2.8 points in the non-recurrent group, and 2.7 in the recurrent group. No statistically significant difference be- tween the two groups was noted in the disc-degeneration score $(p>0.05)$.

Herniated discs were classified as protruded, extruded, or sequestrated based on MRI. There were three protruded (17 $\%)$, seven extruded (39\%), and eight sequestrated discs (44\%) at primary discectomy in the recurrent group. In the non-recurrent group, preoperative MRIs showed that protruded discs were most common in the recurrent group, sequestrated discs were most common. The increased incidence of recurrent disc herniation among patients with previous extruded and sequestrated disc compared to protruded discs was statistically significant $(\mathrm{p}<0.05)$.

Thirty-one patients in the control were cigarette smokers (31\%) and eight patients (44\%) smoked in the recurrent group. Cigarette smokers were more common in the recurrent group than in the control group, although there was no statistically significant association between smoking and the recurrence rate of disc herniation ( $\mathrm{p}>0.05)$.

Radicular pain associated with traumatic events was reported by 26 patients $(27 \%)$ in the control group and 11 patients 
Table 1. Risk factors of recurrent lumbar disc herniation

\begin{tabular}{|c|c|c|c|c|}
\hline & & Control (\%) & Recurrent (\%) & p-value \\
\hline Age (years) & & 46.4yrs & $56.6 y r s$ & $>0.05$ \\
\hline \multirow[t]{2}{*}{ Gender } & Male & 57 & 28 & \multirow[t]{2}{*}{$<0.05$} \\
\hline & Female & 43 & 72 & \\
\hline \multirow{3}{*}{$\begin{array}{l}\text { Level of } \\
\text { discectomy }\end{array}$} & the other levels & 12 & 11 & \multirow[t]{3}{*}{$>0.05$} \\
\hline & $\lfloor 4-5$ & 51 & 61 & \\
\hline & L5-S1 & 37 & 28 & \\
\hline \multirow{5}{*}{$\begin{array}{l}\text { Degree of disc } \\
\text { degeneration } \\
\text { (points) }\end{array}$} & 0 & 1 & 0 & \multirow[t]{5}{*}{$>0.05$} \\
\hline & 1 & 5 & 11 & \\
\hline & 2 & 23 & 28 & \\
\hline & 3 & 55 & 44 & \\
\hline & 4 & 15 & 17 & \\
\hline \multirow{3}{*}{$\begin{array}{l}1^{\text {st }} \text { preoperative } \\
\text { type of herniation }\end{array}$} & Protrusion & 41 & 17 & \multirow[t]{3}{*}{$<0.05$} \\
\hline & Extrusion & 38 & 39 & \\
\hline & Migration & 21 & 44 & \\
\hline Traumatic event & & 27 & 61 & $<0.05$ \\
\hline Smoking status & & 31 & 44 & $>0.05$ \\
\hline
\end{tabular}

Table 2. Pain free interval after $1^{\text {st }}$ operation in recurrent group (Mean 18.7 months)

\begin{tabular}{lc}
\hline \hline & Pain free interval \\
\hline Over 5 years & 1 \\
$3-5$ years & 1 \\
$1-3$ years & 3 \\
$6-12$ months & 13 \\
\hline
\end{tabular}

(61\%) in the recurrent group. Traumatic events occurred at a significantly higher rate in the recurrent group compared to the control group $(\mathrm{p}<0.05)$.

The risk factors for recurrent lumbar disc herniation are summarized in Table 1.

The mean period of relief from pain after primary discectomy was 18.7 months. Thirteen patients underwent reoperation within 1 year after the first operation, three patients from 1 to 3 years, one patient from 3 to 5 years, and one patient more than 5years later (Table 2).

The mean VAS score was 2.3 in the non-recurrent group and, 4.0 in the recurrent group. Clinical outcomes were significantly poorer after repeated discectomy $(p<0.05)$. In addition, according to the modified Macnab criteria, $71 \%$ of patients in recurrent group and $81 \%$ of patients in the control group showed a good or excellent result. The clinical outcomes in the recurrent group were worse than those in the control group (Table 3).

\section{DISCUSSION}

The recurrence of lumbar disc herniation in patients after surgery ranges from 5 to $15 \%$ in various studies, although-
Table 3. Clinical results of recurrent lumbar disc herniation

\begin{tabular}{lccccc}
\hline \hline & VAS score & \multicolumn{4}{c}{ Macnab criteria } \\
\cline { 3 - 6 } & $(p<0.05)$ & Excellent (\%) & Good (\%) & Fair (\%) & Poor (\%) \\
\hline Control & 2.3 & 53 & 28 & 10 & 8 \\
Recurrent & 4 & 28 & 44 & 6 & 22 \\
\hline
\end{tabular}

these studies varied in terms of their patients populations, follow-up periods, the definitions of recurrence, and types of procedures ${ }^{13)}$. In this study, the recurrence rate was $10.1 \%$ at a mean follow-up period of 27.9 months.

The risk factors for recurrent lumbar disc herniation remain controversial. Cinotti et al. ${ }^{6}$ reported that in males, the presence of markedly degenerated discs may be a risk factor for recurrent herniation after microdiscectomy. Suk et al. ${ }^{26}$ reported that young age, the male gender, smoking, and traumatic events are risk factors. According to Kim et al. ${ }^{12)}$ old age and higher BMI are risk factors for recurrence after percutaneous endoscopic lumbar discectomy. However, Swartz et al. ${ }^{28)}$ reported that age, gender, smoking status, level of herniation, and duration of symptoms were not associated with a higher rate of recurrence after partial laminectomy and discectomy. Kim et al. ${ }^{13)}$ also reported that the recurrence rate in young active men was similar to that of the general population.

Cinotti et al. ${ }^{6}$ and Kim et al. ${ }^{12)}$ reported that older patients generally have more degenerated disc-s, which may explain their higher incidence of recurrent herniation. However the degree of disc degeneration was not associated with a higher rate of recurrence in this study, nor was age associated with a higher rate of recurrence after open discectomy.

Unlike Suk et al. ${ }^{26}$ and Cinotti et al. ${ }^{6}$, we found that female gender was a statistically significant risk factor for recurrence. To explain the higher incidence of recurrent herniation in young men, some authors have suggested that the annular incision made at primary surgery makes the operated disc more susceptible to sudden prolapse, particularly under conditions of mechanical overload during sports activity or weightlifting ${ }^{6,13)}$. Because of their body mass, mechanical overload could be relatively higher in women than in men. Without taking into consideration individual occupations or physical activity levels, it may be difficult to conclude that the male gender is a significant risk factor for recurrence. In our opinion, physical factors such as BMI, occupation, and the patient's general medical condition may be important factors influencing the recurrence of disc herniation, although we did not analyze these characteristics because of the lack of relevant date in our clinical records. Although we found female gender to be a significant risk factor, there is limited value in this result because the number of patients with recurrent disc herniation was too small compared to other studies to evaluate 
the female preponderance.

Suk et al..$^{24)}$ and Terhaag et al. ${ }^{27}$ reported that isolated trauma or injury does not seem to play a prominent role in the etiology of disc herniation; this has been reported in only $0.2-10.7 \%$ of adults with documented herniation. Conversely, Cinotti et al. ${ }^{6}$ found that $42 \%$ of patients with recurrent herniation had associated the onset of radicular pain with an isolated injury or a precipitating event; this is higher than in previously reported papers (15-32\%). We found that a traumatic event was associated with recurrent herniation in $61 \%$ of patients, which was much higher than measured by Cinotti et al. ${ }^{6}$ and enough sufficient to consider a traumatic event as a risk factor of recurrence.

Cinotti et al. ${ }^{6}$ found that disc degeneration as seen on preoperative MRI was more severe on average, in patients who developed a recurrent herniation than in the control group. To explain the higher incidence of recurrent herniation in the discs of patients with degenerative changes, Cinotti et al. ${ }^{\text {) }}$ suggested that the healing processes that occur in the outer lamellae after annular injury may lead to a less effective reconstitution of the annulus in markedly degenerated discs, so that a recurrent herniation may be more likely to occur. Dora et al. ${ }^{7}$ reported that a minor degeneration of the disc represents a risk factor for recurrent disc herniation after discectomy. Dora et al. ${ }^{7}$ suggested that Cinotti et al. ${ }^{\text {. }}$ had included patients with abnormalities such as lateral stenosis, narrowed intervertebral foramina, and epidural scarring, and excluded additional confounding factors, such as the morphology of disc herniation, which might have influenced the risk of recurrence. Although we found that the degree of disc degeneration was not associated with a higher rate of recurrence, it is difficult to compare our results with those of Dora et al. ${ }^{7}$ because they used a five-point grading system based on the method of Pfirrmann et al. ${ }^{22)}$ The results of Dora et al. ${ }^{7)}$ combined with our findings suggest that recurrent disc herniation may be more likely to occur in the discs of patients with little or no degenerative changes. A prospective study design based on single shared parameter could more appropriately evaluate this interpretation.

One controversial risk factor for recurrence is the shape of the disc itself ${ }^{28}$. In a study of 531 patients, Morgan-Hough et al. ${ }^{18)}$ reported a revision rate of $7.9 \%$ over a period of 16 years and concluded that a contained disc protrusion was almost three times more likely to require revision surgery compared to extruded or sequestered discs. Kim et al. ${ }^{13)}$ reported that the recurrence rate after open discectomy was significantly higher in the protruded type than other types. They suggested this may be due to the incomplete removal of the herniated disc in protrudedtype injuries ${ }^{13)}$. However, Cinotti et al..$^{6}$ found no difference in the rates of recurrence associated with partial or complete discectomy. Although Suk et al. ${ }^{26}$ asserted that disc shape plays no part in recurrence, Carragee et al. ${ }^{5)}$ prospectively evaluated disc herniation types, the rate of reherniation, and the rate of reoperation, and assessed the type of disc herniation using a penfield dissector, unlike Kim et al. ${ }^{13)}$ and this study, which used MRI images. In the study of Carragee et al. ${ }^{5)}$, patients in a fragment-defect group, who had extruded fragments and massive posterior annular loss, had higher rates of reherniation and reoperation than other groups (a fragment-fissure group, a fragment-contained group, and a group without contained fragments). Carragee et al. ${ }^{5)}$ found that an extruded fragment with massive posterior annular loss had the highest prevalence of recurrent herniation. In this study, recurrent disc herniation developed at a higher incidence with extruded and sequestrated discs than with protruded discs. Similar to Carragee et al..$^{5)}$, we conclude that the annular defects in extruded disc and sequestrated discs are the risk factors for recurrent disc herniation because the intervertebral disc is the most avascular tissue in human body, with low regenerative ability ${ }^{19)}$. When nucleotomy is performed, there is no regeneration of the nucleus pulposus including the annulus fibrosus ${ }^{3,25,27}$. The absence of healing in defective annulus tissue may increase the recurrence rate with extruded and sequestrated types of herniation.

Suk et al. ${ }^{26}$ used the percentage difference between the preoperative and the postoperative pain scores to assess clinical improvement. They reported no significant differences in clinical improvement after revision and the previous surgery ${ }^{26}$ Cinotti et al. ${ }^{6}$ used a 100-point grading system to assess the overall clinical outcome, with 20 points assigned to the severity of pain, 30 to functional status, 20 to patient satisfaction, and 30 to the results of physical examinations. The study found no significant difference in the rate of satisfactory results in recurrent and control groups after primary discectomy and reoperation. In this study, we assessed clinical outcomes using the mean VAS score and modified Macnab criteria and found poorer clinical outcomes in the recurrent group than in the control group. Many institutions recently have reported favorable outcomes after revision surgery for the treatment of relapsed disc herniation ${ }^{21}$. Earlier reports suggesting less favorable outcomes from revision surgery may have been flawed because they included broader post- operative diagnoses such as foraminal stenosis, epidural fibrosis, herniations at other levels, and segmental inst- ability ${ }^{11,21)}$. In this study, no patients had foraminal stenosis or segmental instability at the time of their revision surgery, but the degree of epidural fibrosis was unclear. Repeated surgery in the lumbar spine for recurrent sciatica after a previous discectomy has been associated with poor results when fibrosis is present and the pain-free interval after primary discectomy is shorter than 6-12 months ${ }^{21)}$. Qui- 
mjian and Matrka ${ }^{20)}$ also reported that a longer pain free interval after the first operation is associated with a better prognosis after recurrent lumbar disc herniation. In this study, $72 \%$ of the patients experienced the recurrence of symptoms within 1 year. The clinical outcomes in this study might have been affected by the short pain- free interval before revision surgery, which may have produced the unsatisfactory results of the patients of the recurrent group.

Our study had several important limitations. One of the most important factors influencing the recurrence rate of disc herniation is the thoroughness of follow-up and the number of cases $^{17}$, and our study was limited by a high rate of follow-up loss, an insufficient follow-up period, and a small number of patients.; these factors may have prevented us from detecting significant differences among the groups and from evaluating the risk factors for recurrent herniation and clinical outcomes. In addition, our study was limited by a lack of accurate hospital records to review retrospectively. Finally, we did not include other factors such as diabetes, obesity, and hospitalizations, which might be more applicable to clinical parameters ${ }^{17,26,28)}$.

\section{CONCLUSION}

The recurrence rate of lumbar disc herniation was $10.1 \%$ in our patients. Fewer patients had satisfactory results after reoperation for recurrent radicular pain than after the primary operation.

The risk factors for recurrent lumbar disc herniation in this study were female gender, the type of disc herniation (extruded, sequestrated), and traumatic events.

\section{REFERENCES}

1. Andrews DW, Lavyne MH: Retrospective analysis of microsurgical and standard lumbar discectomy. Spine (Phila Pa 1976) 15:329-35, 1990

2. Babar S, Saifuddin A: MRI of the post-discectomy lumbar spine. Clin Radiol 57:969-981, 2002

3. Bayliss MT, Urban JP, Johnstone B, Holm S: In vitro method for measuring synthesis rates in the intervertebral disc. J Orthop Res 4:10-17, 1986

4. Boden SD, Riew KD, Yamaguchi K, Branch TP, Schellinger D, Wiesel SW: Orientation of the lumbar facet joints: association with degenerative disc disease. J Bone Joint Surg Am 78:403411, 1996

5. Carragee EJ, Han MY, Suen PW, Kim D: Clinical outcomes after lumbar discectomy for sciatica: the effects of fragment type and annular competence. J Bone Joint Surg Am 85-A:102-108, 2003

6. Cinotti G, Roysam GS, Eisenstein SM, Postacchini F: Ipsilateral recurrent disc herniation: a prospective, controlled study. J
Bone Joint Surg Br 80:825-832, 1998

7. Dora C, Schmid MR, Elfering A, Zanetti M, Hodler J, Boos $\mathrm{N}$ : Lumbar disk herniation: do MR imaging findings predict recurrence after surgical diskectomy? Radiology 235(2):562-567, 2005

8. Eismont FJ, Currier B: Surgical management of lumbar intervertebral-disc disease. J Bone Joint Surg Am 71:1266-1271, 1989

9. Graver V, Haaland AK, Magnaes B, Loeb M: Seven-year clinical follow-up after lumbar disc surgery: results and predictors of outcome. Br J Neurosurg 13:178-184, 1999

10. Horton WC, Daftari TK: Which disc as visualised by magnetic resonance imaging is actually a source of pain? a correlation between magnetic resonance imaging and discography. Spine (Phila Pa 1976) 17(6 Suppl):164-171, 1992

11. Jung YS, Choi HJ, Kwon YM: Clinical Outcome and In fluencing Factor for Repeat Lumbar Discectomy for Ipsilateral Recurrent Lumbar Disc Herniation. Korean J Spine 9(1):1-5, 2012

12. Kim JM, Lee SH, Ahn Y, Yoon DH, Lee CD, Lim ST: Recurrence after successful percutaneous endoscopic lumbar discectomy. Minim Invasive Neurosurg 50:82-85, 2007

13. Kim MS, Park KW, Hwang C, Lee YK, Koo KH, Chang BS, et al: Recurrence rate of lumbar disc herniation after open discectomy in active young men. Spine 34(1):24-29, 2009

14. Masaryk TJ, Ross JS, Modic MT, Boumphrey F, Bohlman H, Wilbert G: High-resolution MR imaging of sequestered lumbar intervertebral disks. AJR Am J Roentgenol 150:1155-1162, 1988

15. Mirowitz SA, Shady KL: Gadopentetate dimeglumine? enhanced MR imaging of the postoperative lumbar spine: comparison of fat-suppressed and conventional T1-weighted images. AJR Am J Roentgenol 159:385-389, 1992

16. Modic MT, Steinberg PM, Ross JS, Masaryk TJ, Carter JR: Degenerative disk disease: assessment of changes in vertebral body marrow with MR imaging. Radiology 166:193-199, 1988

17. Mobbs RJ, Newcombe RL, Chandran KN: Lumbar discectomy and the diabetic patient: incidence and outcome. J Clin Neurosci 8:10-13, 2001

18. Morgan-Hough CV, Jones PW, Eisenstein SM: Primary and revision lumbar discectomy. A 16-year review from one centre. J Bone Joint Surg Br 85:871-874, 2003

19. Ochi M, Uchio Y, Tobita M, Kuriwaka M: Current concepts in tissue engineering technique for repair of cartilage defect. Artif Organs 25:172-179, 2001

20. O'Sullivan MG, Connolly AE, Buckley TF: Recurrent lumbar disc protrusion. Br J Neurosurg 4:319-325, 1990

21. Papadopoulos EC, Girardi FP, Sandhu HS, Sama AA, Parvataneni HK, O'Leary PF, et al: Outcome of revision discectomies following recurrent lumbar disc herniation. Spine (Phila Pa 1976) 31(13):1473-1476, 2006

22. Pfirrmann CW, Metzdorf A, Zanetti M, Hodler J, Boos N: Magnetic resonance classification of lumbar intervertebral disc degeneration. Spine (Phila Pa 1976) 26:1873-1878, 2001

23. Quimjian JD, Matrka PJ: Decompression laminectomy and lateral spinal fusion in patients with previously failed lumbar spine surgery. Orthopedics 11:563-569, 1988

24. Ross JS: MR imaging of the postoperative lumbar spine. Magn Reson Imaging Clin N Am 7:513-524, 1999

25. Sato M, Asazuma T, Ishihara M, Kikuchi T, Kikuchi M, Fujikawa K: An experimental study of the regeneration of the inter- 
vertebral disc with an allograft of cultured annulus fibrosus cells using a tissue-engineering method. Spine (Phila Pa 1976) 28: 548-553, 2003

26. Suk KS, Lee HM, Moon SH, Kim NH: Recurrent lumbar disc herniation: result of operative management. Spine (Phila Pa 1976) 26:672-676, 2001

27. Sumida K, Sato K, Aoki M, Matsuyama Y, Iwata H: Serial chan- ges in the rate of proteoglycan synthesis after chemonucleolysis of rabbit intervertebral discs. Spine (Phila Pa 1976) 24:10661070, 1999

28. Swartz KR, Trost GR: Recurrent lumbar disc herniation. Neurosurg Focus 15:E10, 2003

29. Terhaag D, Frowein RA: Traumatic disc prolapses. Neurosurg Rev 12(Suppl 1):588-594, 1989 